\title{
A Review of COVID-19: Data Obtained from Chest CT Imaging and RT-PCR
}

\author{
Hossein Akbari-Lalimi ${ }^{1}$, Somayyeh Seyedi ${ }^{1}$, Hadi Akbari-Zadeh ${ }^{1}$, Milad Najafzadeh ${ }^{1}$, Alireza \\ Montazerabadi ${ }^{1}$, Behzad Aminzadeh ${ }^{2}$ and Farzaneh Ghorbani ${ }^{1,3,{ }^{*}}$ \\ ${ }^{1}$ Department and Research Center of Medical Physics, Mashhad University of Medical Sciences, Mashhad, Iran \\ ${ }^{2}$ Department of Radiology, Faculty of Medicine, Mashhad University of Medical Sciences, Mashhad, Iran \\ ${ }^{3}$ Student Research Committee, Mashhad University of Medical Sciences, Mashhad, Iran \\ "Corresponding author: Student Research Committee, Mashhad University of Medical Sciences, Mashhad, Iran. Email: ghorbanifme@gmail.com
}

Received 2020 July 03; Revised 2021 April 07; Accepted 2021 April 14.

\begin{abstract}
Context: This review aimed to investigate the computed tomography (CT) imaging features of coronavirus disease 2019 (COVID-19). Evidence Acquisition: Bilateral lung involvement (70\%), peripheral distribution (67.5\%), multifocal involvement (67.3\%) and ground glass opacity (66.1\%) were observed in most infected patients.

Results: Evaluation of the number of lobes involved in infected patients showed no abnormalities in 7.2\% (85/1177) of the patients. Regarding the performance of reverse transcription-polymerase chain reaction (RT-PCR) and chest CT scan, the sensitivity of RT-PCR and chest CT imaging was estimated at 70\% (925/1311) and 89\% (6605/7396) upon admission, respectively; nevertheless, the sensitivity of CT imaging increased as the time from the symptom onset increased. The CT image acquisition parameters affecting image quality and patient dose were also discussed. Studies suggested that these factors should be adjusted according to the disease stage. Based on our findings, sensitivity was adequately high eight days after the onset of symptoms.

Conclusion: Therefore, there is no need for high-resolution chest CT scan after this interval. Using ImpactDose software, the mean effective doses were 4.38 and $5.71 \mathrm{mSv}$ in male and female groups, respectively. The risk of cancer was $36 \%$ higher in females than males, as shown by PCXMC program.
\end{abstract}

Keywords: COVID-19, SARS-CoV-2, CT, RT-PCR, Sensitivity, Effective Dose, Pneumonia

\section{Context}

The infection caused by SARS-CoV-2, called coronavirus disease 2019 (COVID-19) by the World Health Organization (WHO), was first reported in December 2019 in Wuhan, China. On January 30, 2020, the WHO Emergency Committee warned that the rate of this infection was on the rise in China and other countries. Finally, the WHO declared it as a pandemic on March 11, 2020 (1). COVID19 has a highly contagious nature and is easily transmitted from one individual to another, leading to a respiratory system dysfunction (2). According to the sixth trial of Chinese government guidelines for COVID-19 management, reverse transcription-polymerase chain reaction (RT-PCR) and gene sequencing for respiratory or blood specimens are the reference tools for diagnosing COVID19 and hospitalization of infected cases. Nonetheless, the non-negligible rate of false-positive (FP) results undermines the reliability of these tests (3).
Other medical tools that are used to monitor the disease progression include chest radiography and computed tomography (CT) scan, as the virus targets the respiratory system (4). However, today, the importance of CT imaging is being discussed as a more efficient diagnostic and tracking tool as opposed to RT-PCR $(2,5)$. This paper reviewed the efficiency of CT imaging in diagnosis and tracking of COVID-19 progression compared to RT-PCR and to study the CT imaging features of this disease. Moreover, the CT image acquisition parameters, affecting image quality and patient dose, were discussed to emphasize the important contribution of CT scan to disease diagnosis and control.

\section{Diagnosis of COVID-19}

The timely diagnosis of a disease can play an important role in its control and treatment. Diagnosis becomes even more important in contagious diseases, such as COVID-19 
due to its high prevalence and absence of symptoms several days after infection. Besides, by rapid diagnosis and isolation of the patient, the spread of the disease can be prevented $(3,4)$. Various studies have used laboratory tests, chest imaging, and RT-PCR of respiratory secretions for diagnosis of this infection. Also, isolation of COVID-19 and genetic sequences can be used for detecting the virus (6).

\subsection{RT-PCR Assay}

Since January 11, 2020, upon isolation of the viral strain of COVID-19 and establishment of its whole genome sequences, RT-PCR has been used to diagnose this infection. Soon later, contact history, clinical manifestations, and imaging evidence of pulmonary lesion were considered as the main factors for disease identification; however, RT-PCR is still being used for some suspected cases (7-10). To perform this test, respiratory secretions are obtained from the bronchoalveolar lavage, endotracheal aspirate, nasopharyngeal swab, or oropharyngeal swab (1). This test has a high specificity for COVID-19 (11). In other words, it can identify the virus subgroup, as Middle East respiratory syndrome (MERS)-CoV, SARS-CoV, and SARS-CoV-2 have similar clinical symptoms and effects on the respiratory system (1, 3,12 ).

Despite its advantages, RT-PCR has some limitations. The low sensitivity of RT-PCR for identification of COVID19 has been reported in various studies $(1,3)$. The timeconsuming nature of this test and insufficient RT-PCR kits are other major issues during this pandemic. Likewise, lack of standard operating procedures for SARS-CoV-2 nucleic acid detection, improper sampling, insufficient viral material in the specimen (depending on the stage of the disease), sample handling, storage, and processing, and kit efficiency should be addressed, as well (4). Previous SARS studies show that RT-PCR lacks sensitivity within the first five days of the disease (13). Therefore, when the specimen tests are negative, the possibility of a false-negative (FN) result should be considered in the context of a patient's recent exposure and clinical signs and symptoms consistent with SARS-CoV-2 infection $(1,3,5)$.

\subsection{CT Imaging}

CT imaging, as a routine imaging tool which is available and relatively easy to perform, can be used to screen patients for rapid diagnosis and is more effective in detection of pneumonia caused by SARS-CoV-2. The majority of previous studies have reported that high-resolution computed tomography (HRCT) of the chest can identify infection in the respiratory system of COVID-19 patients $(7,11)$.

\subsubsection{CT Imaging Features}

According to a study by Wu et al., pneumonia was the main finding in chest CT images $(76 / 80,95 \%)$ at 7 \pm 4 days after the onset of COVID-19 (5). Several studies have reported ground glass opacities (GGOs) or mixed GGOs, as well as patchy consolidations (sometimes oval rather than patchy) in the patients' lungs after infection $(3,7,8,14)$. In a study by Xie et al., the CT imaging features attributed to COVID-19 included GGOs, consolidations, mixed GGO with consolidation, traction bronchiectasis, bronchial wall thickening, reticulation, subpleural bands, and vascular enlargement (15). The percentages of CT image features are presented in Table 1. The most common pattern was GGO in CT scans. The percentage of each feature was calculated based on the ratio of all patients having a given feature to all patients investigated for that feature in all studies of interest (1-3, 5, 8, 9, 11, 12, 14-54).

Atypical CT manifestations can be particularly seen in the elderly or severe cases, including multiple patchy consolidations or large patches of consolidation in both lungs, with limited grid-like or honeycomb-shaped interlobular septal thickening, especially in the middle and lower lobes; single or multiple solid nodules; or consolidated nodules in the center of lobule, surrounded by GGOs $(8,15,40,55)$.

The location of lesions is another interesting topic in COVID-19 management. A recent study reported that lesions mostly occurred in the peripheral area under the pleura, although they might extend toward the center in larger lesions, as observed in severe and critically severe cases. In these patients, 4 - 5 lobes, and most significantly, bilateral lower and upper lobes, were mostly involved. Nonetheless, the 4- to 10-day follow-up CT scans indicated improvements in the lungs, including the reduced extent of lesions, decreased density, and formation of fibrotic stripes (4). Figure 1 summarizes the lesion sites in the lungs, according to various studies reporting the lesion sites in detail.

As can be seen in Figure 2, bilateral lower lobes and the right middle lobe were the most and the least infected lung areas, respectively. The percentages of infected cases with different numbers of lobes involved are shown in Figure 2. Patients with the involvement of all lobes comprised the largest group. According to Figure 2, only 7.2\% (85/1177) of the patients had no involved lobes, and $35 \%$ of the infected population had 4 - 5 lobes involved, indicating that COVID19 quickly spreads over the lung tissue. The total number of patients was 1177 in studies investigating the number of involved lobes (Figure 2).

Despite the similarity of COVID-19 symptoms and other types of coronavirus, definite epidemical history-taking 


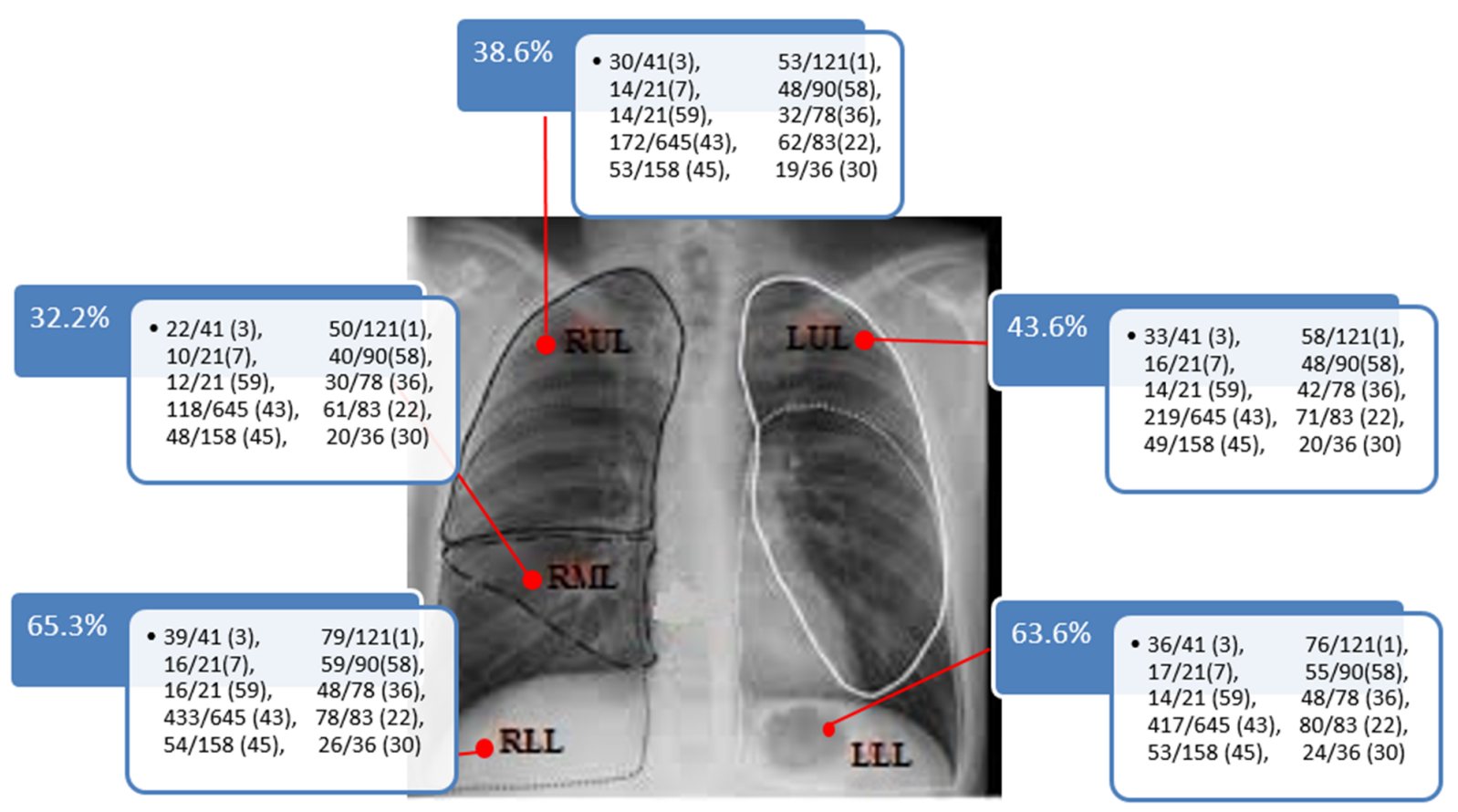

Figure 1. The percentage of patients with the specified lobe involvements in different studies $(1,4,7,22,30,36,43,45,56,57)$. For each lobe, the number of infected cases is separately presented in each study.

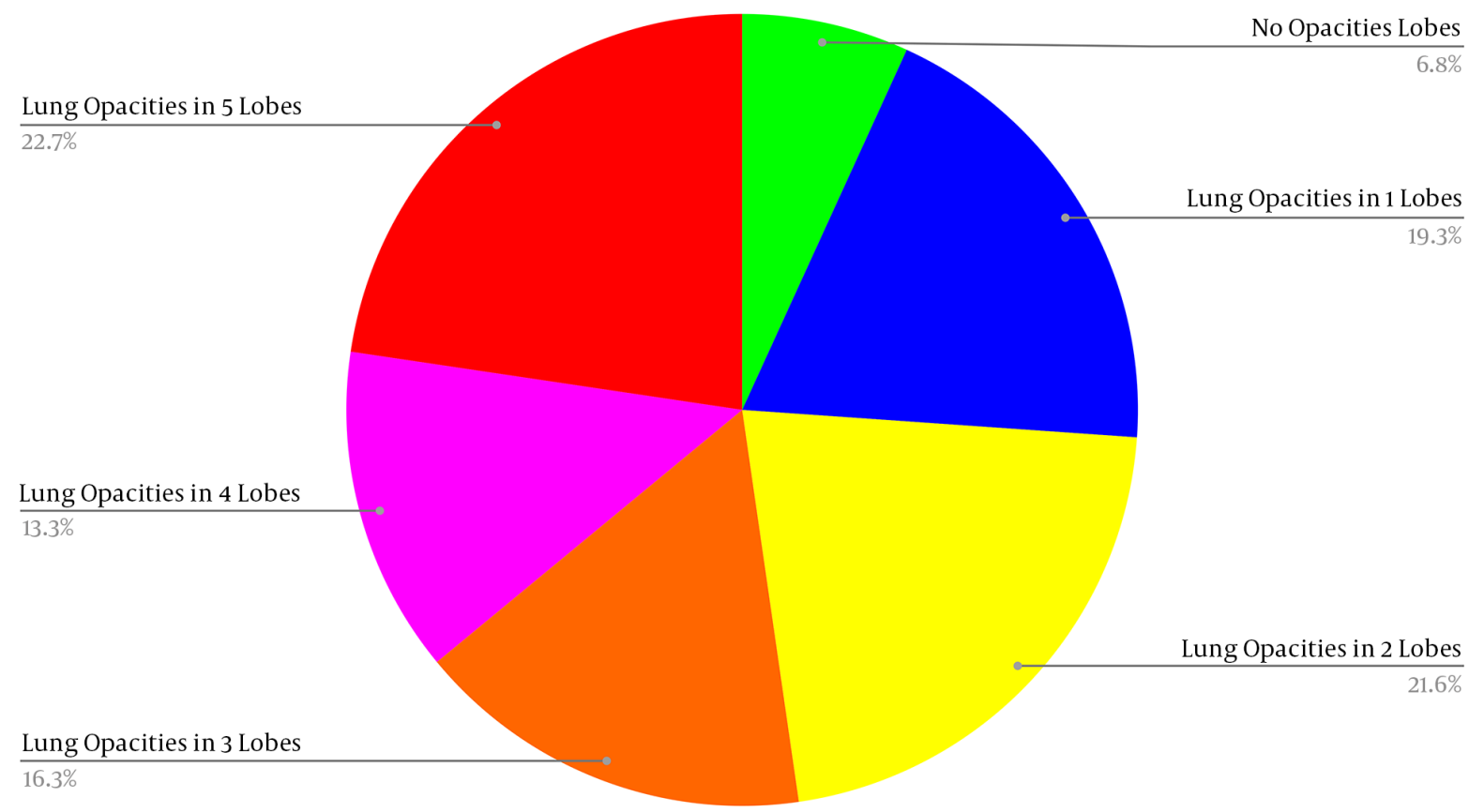

Figure 2. The frequency percentages of patients with the specified lobe involvements $(1,4,6,10,11,19,23,26,27,33,36,39,41,43,45,52,54-58)$. 


\begin{tabular}{|c|c|}
\hline Features & Percentage \\
\hline Bilateral lung involvement & 70.0 \\
\hline Peripheral distribution & 67.5 \\
\hline Multifocal involvement & 67.3 \\
\hline GGO & 66.1 \\
\hline Mixed GGO and consolidation & 49.9 \\
\hline Thickened interlobular septa & 43.3 \\
\hline Rounded opacities & 41.8 \\
\hline Air bronchogram & 41.5 \\
\hline Linear opacities & 39.1 \\
\hline Reticular pattern & 37.7 \\
\hline Multiple mottling and GGO & 30.0 \\
\hline Central + peripheral distribution & 27.8 \\
\hline Consolidation & 26.4 \\
\hline Subpleural line & 24.6 \\
\hline Spider web sign & 22.3 \\
\hline Crazy-paving pattern & 19.1 \\
\hline Bronchiectasis & 18.5 \\
\hline Pulmonary nodules & 16.0 \\
\hline Unifocal involvement & 14.1 \\
\hline Bronchial wall thickening & 13.8 \\
\hline Normal CT & 9.9 \\
\hline Underlying pulmonary fibrosis & 9.4 \\
\hline Diffuse distribution & 8.8 \\
\hline Lymphadenopathy & 6.4 \\
\hline Pericardial effusion & 5.5 \\
\hline Pleural effusion & 4.9 \\
\hline "Reverse halo" sign & 3.8 \\
\hline Central distribution & 3.7 \\
\hline Enlarged mediastinal nodes & 2.9 \\
\hline Lung cavitation & 0 \\
\hline
\end{tabular}

Abbreviation: GGO, ground glass opacities.

and viral nucleic acids can be used to differentiate between pneumonia caused by COVID-19 and pneumonia caused by other viruses, such as influenza virus, parainfluenza virus, adenovirus, and SARS-CoV, or other microorganisms, including Mycoplasma, chlamydia, and bacteria. Large diffuse lesions of GGO in both lungs, accompanied by interlobular septa, are common in these infections (4).

Liu et al. suggested that in the clinical treatment of COVID-19, patients with a small amount of pleural effusion or pleural thickening may have a serious condition and need to be actively treated. Besides, lung lesions may still exist after the negative detection of SARS-CoV-2 nucleic acids, and follow-up may be needed (14). Pulmonary thromboembolism (PTE) caused by COVID-19, with a prevalence of up to $40 \%$ based on CT angiography, has been reported in several studies $(59,60)$. In this regard, Cavagna et al. investigated 101 patients infected with COVID-19 and found that PTE involved segmental and subsegmental venous systems more commonly and was more likely to be bilateral or have a right lobe involvement pattern. They also reported that consolidations were more dominant in the PTE-involved lung segments as compared to other segments (61). Besides, Zitzmann et al. reported that subpleural consolidations were related to PTE. They suggested that patients with a high D-dimer level and subpleural consolidations should be examined in terms of PTE (61).

\subsubsection{Performance of CT Imaging for Diagnosis of COVID-19}

The higher sensitivity and specificity of a test compared to other available tests are the main factors in test selection, as they are associated with more reliable results and less uncertainty of diagnosis. However, other factors, such as cost, risks, and the time needed for preparing the results, should be also considered $(1,7,11,23)$. Although chest CT findings are non-specific for COVID-19 detection, some studies suggested that $\mathrm{CT}$ could be used as an alternative diagnostic tool $(7,18,47)$. Recent reports show that chest CT imaging can demonstrate pneumonia, even if multiple RT-PCR tests of nasopharyngeal or throat swabs are negative (3). Since several factors, resulting in FN values, can intervene in the RT-PCR results $(1,3,8,56)$, and the CT results are evaluated based on RT-PCR, it can be concluded that the FP values of CT are overestimated, and subsequently, the specificity of this tool is underestimated (3). Considering the mentioned advantages and disadvantages of RT-PCR and CT imaging, their comparison for COVID-19 diagnosis is summarized in Table 2.

Despite the CT imaging advantages, this modality can increase the risk of cancer due to the use of ionizing radiation. Based on the International Society of Ultrasound in Obstetrics and Gynecology (ISUOG) guidelines, chest imaging, especially CT scan, is essential for evaluating the clinical status of pregnant women with COVID-19. The radiation dose to the fetus is 0.01-0.66 mGy from a single chest CT scan, which is negligible without any complications (63).

In a study by Ai et al., RT-PCR was used as the reference. The results of RT-PCR were negative in $35 \%$ of patients diagnosed with COVID-19 based on CT scans; the high number of FP values in CT was the reason for this finding. However, it should be noted that the sensitivity of RT-PCR was 


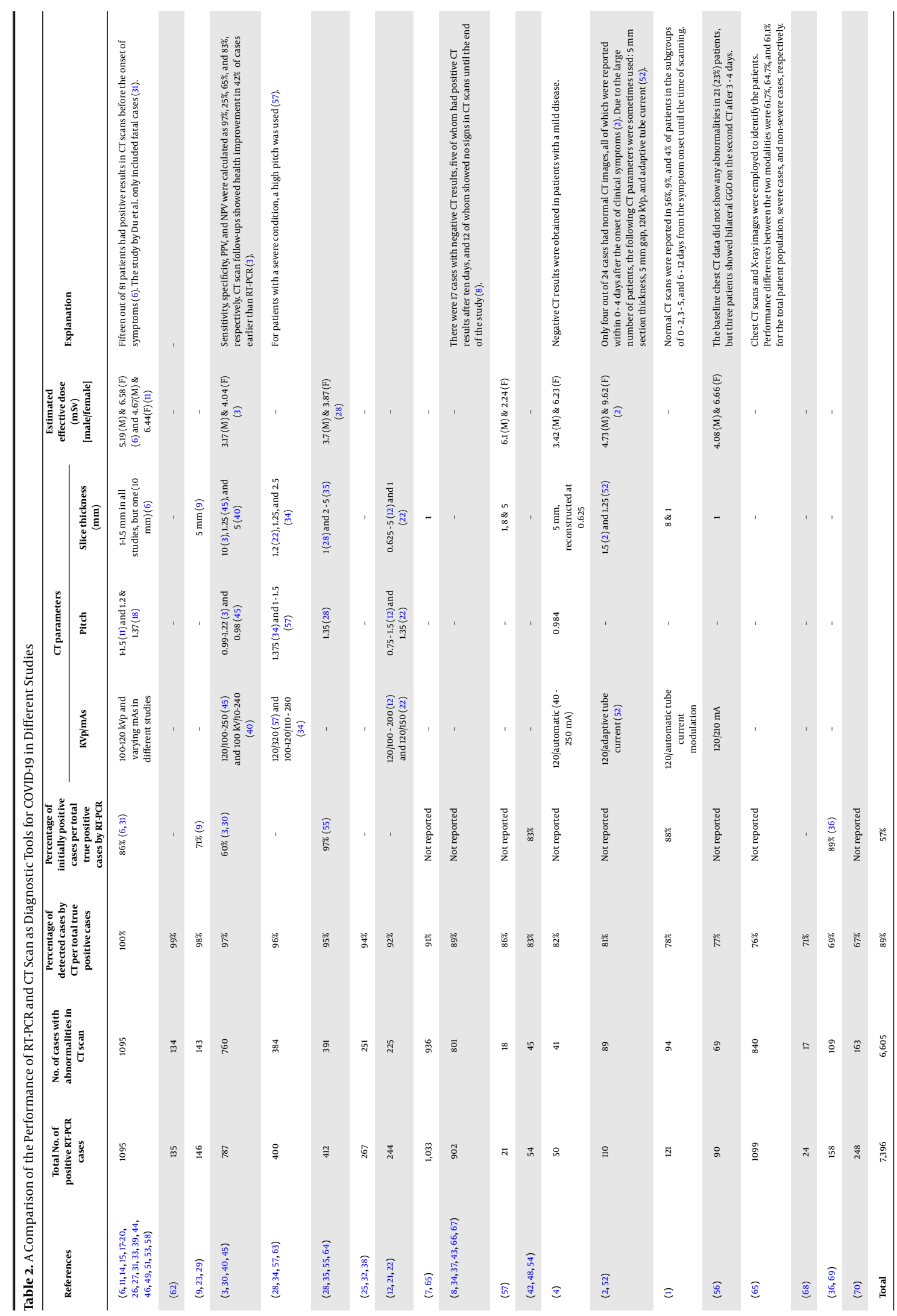


lower than expected. Besides, the negative predictive values (NPVs) indicated that the rate of misdiagnosis of patients as healthy cases by CT was 17\% (3). Ai et al. also found that 147 out of 308 (48\%) patients with FP values were more likely to be infected. Based on this assumption, the sensitivity, specificity, and positive predictive value (PPV) would be $97.5 \%, 64 \%$, and $93 \%$, respectively. In other words, the FP values of CT imaging were higher than the real FP values based on the first RT-PCR. With RT-PCR repetition and patient follow-up, it was found that only $7 \%$ of cases with positive CT results had negative RT-PCR results. As explained earlier, the FP value of CT was estimated to be $35 \%$, based on the first results of RT-PCR without this correction.

According to studies described in Table 2, the percentage of non-detected cases by CT was 10\% (692/6867). This result might be due to the fact that the patients were probably in the early stages of the disease, and subsequently, there were no lesions in the lung tissue. Similarly, a study by Guan et al. showed that chest CT scan could identify $91.3 \%$ of patients with a severe condition and $73.3 \%$ of patients with a non-severe condition (65). Similar results have been reported in some other studies $(1,2,4)$, summarized in Table 2. These findings demonstrated that COVID19 had a gradual impact on the lung tissue. Observation of these lung alterations relatively depended on the time interval between the symptom onset and $\mathrm{CT}$ image acquisition, as illustrated in Figure 3. Similar results were obtained for 73 SARS-infected cases, as the CT results were normal in patients with mild symptoms (13).

As can be seen in Figure 3, the percentage of patients with normal CT scans decreased as the time interval increased from the onset of symptoms. In other words, the probability of COVID-19 manifestation on CT scan was low before the symptom onset or in early stages of the disease, although it increased due to gradual disease progression. It should be noted that the middle point of this interval was considered as the time of $\mathrm{CT}$ image acquisition in studies reporting the intervals between the symptom onset and CT image acquisition, whereas in other studies, the mean or median of intervals was considered as the time of CT image acquisition. In contrast, abnormal CT findings with the initial FN results of RT-PCR have been documented $(1,3,30)$. Also, Shi et al. reported that CT images could identify early changes in the respiratory system of asymptomatic cases because of COVID-19 (6).

In previous studies examining the sensitivity of RTPCR, the rate of positive results was $70 \%$ in the first test. Nonetheless, CT could identify $93 \%$ of cases in the first exposure, as reported in multiple studies $(1,3,6,7,15,16,37,39$, $42,65,72-75$ ) (Table 2). Interestingly, a similar percentage
(67\%) was reported for identification of SARS for the first time using RT-PCR (74). Unlike RT-PCR that can only diagnose COVID-19, CT imaging can diagnose the infection and show the disease progression. CT can also demonstrate whether there are still lesions in the lung tissue in the absence of clinical symptoms. Therefore, it is suggested that chest $\mathrm{CT}$ follow-ups be performed after patient discharge and during prescribed drug consumption intervals $(3,7,14$, 26, 28).

Artificial intelligence (AI) technologies, such as deep learning, can contribute to image feature extraction and subsequently, to rapid clinical diagnosis. Wang et al. assessed the performance of deep learning algorithms for COVID-19. A total of $453 \mathrm{CT}$ images of definite cases were included in their study. The results demonstrated that the algorithm provided an accuracy of $82.9 \%$, specificity of $80.5 \%$, and sensitivity of $84 \%$ as compared to RT-PCR. The measured sensitivity is compatible with the mean sensitivity that we calculated based on different studies reviewed. Therefore, it can be concluded that assessment of image features using deep learning algorithms significantly facilitates timely and accurate diagnosis of the disease, particularly when dealing with a heavy workload (76).

\subsubsection{Acquisition Parameters}

Image quality is one of the most important factors in the interpretation of CT images and has a direct impact on clinical diagnosis. Different acquisition parameters influence the quality of CT images; therefore, the results of CT depend on the protocols, particularly in the early stages of COVID-19. However, even by using proper parameters, some studies reported the negative results of chest CT imaging when the RT-PCR results were positive $(8,57$, 65). Their findings revealed that a normal chest CT scan could not be interpreted as the absence of infection in suspected cases. Moreover, the possibility of FN results of RTPCR should be considered regarding the patient's exposure history, typical CT imaging features, the presence of clinical symptoms, and dynamic changes similar to COVID-19 (3).

Wong et al. conducted a study to investigate the lesion size in the lung tissue due to SARS (13). They acquired two CT images of 149 SARS-infected patients. The first scans were the conventional CT scans of the thorax with a 7-mm slice thickness and a pitch of 1.5 . The second scans were acquired via HRCT with a 1-mm slice thickness and a 6-mm gap for a better assessment; the $\mathrm{kVp}$ and $\mathrm{mAs}$ were almost the same in the two CT series. The results revealed that small lesion sizes were better observed in small slice thicknesses. Therefore, it can be concluded that for COVID-19 di- 


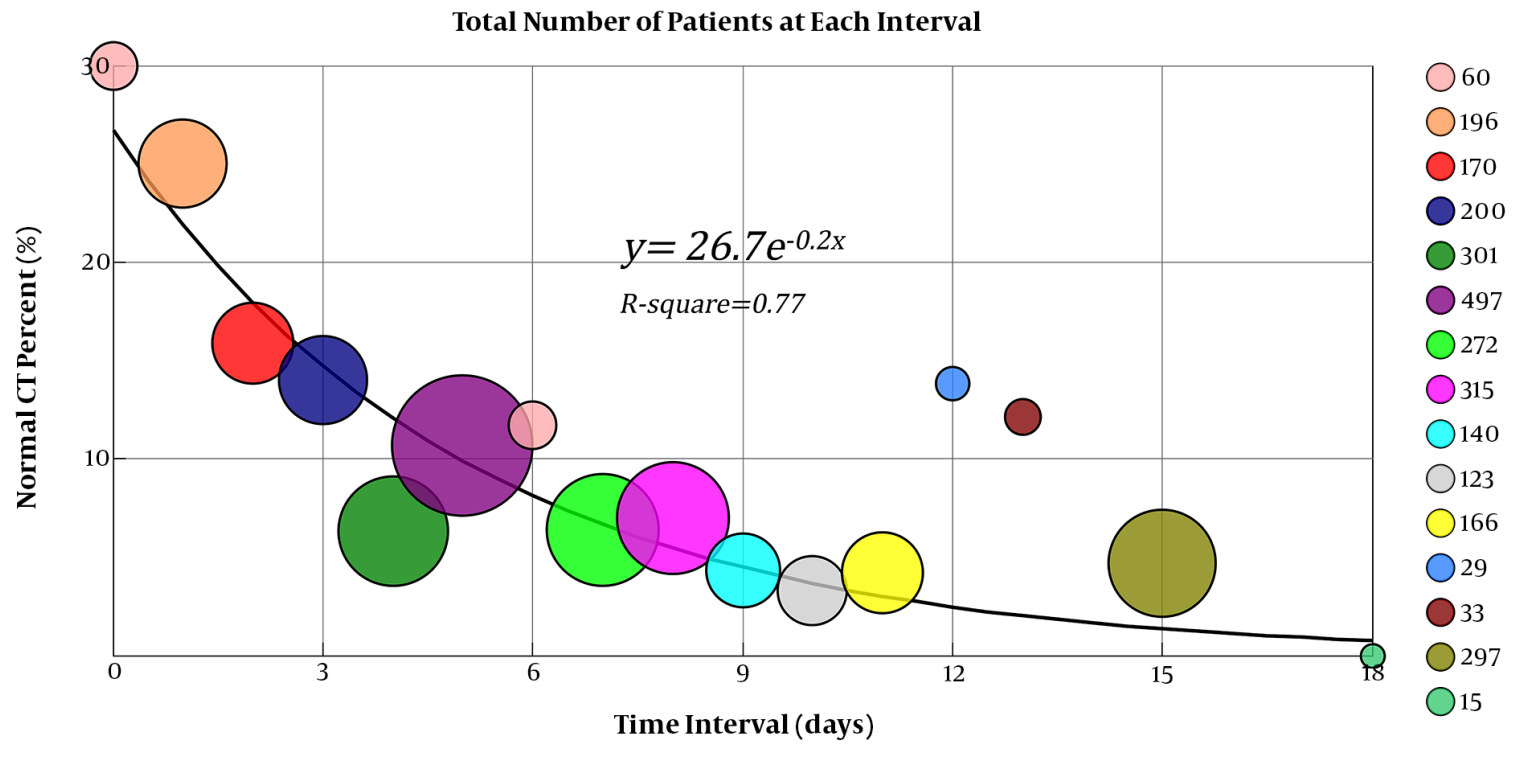

Figure 3. The percentage of COVID-19 patients with normal CT results in each interval between the onset of symptoms and CT image acquisition. Each circle represents the number of patients with normal CT results in a specific interval. For instance, patients with CT scans acquired at 11,13, and 18 days after the onset of symptoms were combined into one group; there were no patients with normal CT results in this group. The total number of patients with the reported time interval between the symptom onset and CT image acquisition was $2,751(1,2,5-10,15,17,22-27,29-34,37,39,40,42,44,46,51-54,58,71,72)$.

agnosis, a smaller slice thickness and a lower pitch were associated with the higher sensitivity of CT scans, as there are similar characteristics between COVID-19 and SARS.

Moreover, in a study by Bernheim et al., the patients were scanned with different slice thicknesses. However, it was not discussed what slice thickness was used for patients with normal CT scans; therefore, we could not discuss this study. Nonetheless, it can be concluded that CT scans have a lower sensitivity in patients who are in early stages of the disease (1). A similar conclusion was made by Xu et al.; the imaging parameters are shown in Table 2. The width/level of $2000 /-600 \mathrm{HU}$ for the lung window and 350/40 HU for the mediastinal window were adjusted in their study, as well (4). The CT results were normal for all patients with mild symptoms. Since slice thickness plays a key role in determining spatial resolution and image reconstruction, one of the reasons for negative CT results could be the large slice thickness, causing problems in image reconstruction. However, the disease might not cause lesions in some cases; these cases had normal CT results, even in HRCT (4), and RT-PCR was crucial for diagnosis.

Besides, Pan et al. investigated the CT scan changes of COVID-19 during the recovery process (2). The initial CT images of 4/21 (19\%) patients did not indicate any lung lesions in the time interval of $2 \pm 2$ days after the symptom on- set. However, lung involvement increased within ten days after the symptom onset, followed by a decreasing trend thereafter. According to the disease classification by Bernheim et al. (1), in which patients are categorized into three groups of early (0-2 days), intermediate(3-5 days), and late (6 - 12 days) according to the interval between the initial onset of symptoms and the subsequent chest CT scan, patients in the study by Pan et al. were in the early stage of the disease. However, the sensitivity of CT scan was reported to be $95 \%$, which is higher than the percentage reported by Bernheim et al. for this group. The image acquisition parameters were as follows: slice thickness of $1.5 \mathrm{~mm}$ with an increment of $1.5 \mathrm{~mm}$ and a repetitive reconstruction algorithm with a B70F kernel and a B30F lung kernel; therefore, the smaller slice thickness could be the cause of sensitivity differences between these two studies. It is recommended to perform CT imaging with small slice thicknesses to acquire chest images in cases with mild symptoms.

Moreover, Wu et al. reported that the average time between hospital admission and CT imaging was $7 \pm 4$ days (5). The disease was categorized into intermediate and late stages according to the classification by Bernheim et al. (1). In other words, the patients had symptoms, including pneumonia, as indicated by CT with high sensitivity. It should be noted that they used a B80f reconstruction 
kernel (a sharp kernel) and a high-resolution algorithm in their study. It is suggested to conduct CT imaging with a high pitch to accelerate CT image acquisition and decrease the workload for patients with severe respiratory problems. Also, for these patients, it is possible to increase the slice thickness, which is most beneficial during this epidemic due to high requests for CT scans.

Previous studies have indicated that a chest CT examination delivered a dose, which was 100 - 500 times higher than conventional chest X-ray on average, thereby increasing the risk of cancer $(73,75)$. Since radiation exposure in CT imaging can increase the risk of cancer and CT scans are commonly performed in patients with COVID-19, a compromise between image quality and effective dose seems necessary (77). Besides, in this review, using the International Commission on Radiological Protection (ICRP013) weighting factors and ImpactDose v. 2.2 (CT Imaging GmbH, Erlangen, Germany), we evaluated the effective dose, based on the image acquisition parameters mentioned in several studies $(2-6,11,56,57)$. Different parameters, including gender, age, $\mathrm{kVp}$, mAs, scan length, CT dose index (CTDI), scanner type, and patient's anterior and posterior lengths, were considered in the estimations. Also, PCXMC v. 2 (STUK, Helsinki, Finland) was used (78) to calculate the cancer risk, using categorized tables in the National Academy of Sciences Biologic Effects of Ionizing Radiation (BEIR) VII report.

For effective dose estimations, the image acquisition parameters of 1,420 patients, including 667 men and 753 women, were examined. The mean age of this population was $47 \pm 3.8$ years. In the male group, the mean effective dose was measured to be $4.38 \mathrm{mSv}$. Also, most effective doses were reported for the heart (13.54 mSv), lungs (12.93 $\mathrm{mSv})$, and thyroid (9.8 $\mathrm{mSv})$. On the other hand, in the female group, the mean effective dose was $5.71 \mathrm{mSv}$. Most effective doses were estimated in the heart (13.27 mSv), lungs (11.33 mSv), breasts ( $8.23 \mathrm{mSv}$ ), and thyroid (6.63 mSv), respectively.

Additionally, the cancer risk was estimated to be higher in the female group compared to the male group (36\%) in this review, which is compatible with previous studies in the literature (79). Therefore, due to the COVID-19 outbreak and the necessity of repeated CT image acquisition at admission or in follow-ups, it is highly recommended to optimize the image acquisition parameters. Different studies have investigated the influential parameters in the effective dose. A large slice thickness, high pitch, automated tube current modulation technique, iterative reconstruction algorithm, precise adjustment of scan range, and correct patient positioning are the main factors in dose reduc- tion. However, they should be adjusted to achieve a reasonable image quality $(78,80)$.

\section{Conclusion}

COVID-19 imposes a serious economic burden on governments and healthcare systems, including heavy workloads and high costs of diagnosis and treatment due to its rapid outbreak in a limited period. In the absence of effective treatments, the most applicable strategy to deal with the SARS-CoV-2 epidemic is appropriate and fast screening of suspected cases. By using this strategy, not only infected cases are quarantined and receive proper medical care, but also the possibility of disease spread decreases significantly. However, since $89 \%$ of infected cases are hospitalized after five days of disease onset (72), identification and isolation of suspected cases in the earlier stages of the disease seems difficult.

In CT imaging as a diagnostic tool, spatial resolution plays a decisive role in finding lesions caused by COVID-19. Besides image quality, another important aspect of CT scan is the effective dose; therefore, a compromise between image quality and effective dose seems necessary. Slice thickness and pitch were effective parameters in both spatial resolution and the dose received by the patients. Based on various studies, a small slice thickness and a pitch $<1$ led to a better detection of lesions in patients in the early stage of the disease. Along with disease progression, and subsequently, lesion enlargement, a larger slice thickness and pitch $>1$ could be used ( 81 ).

For dose estimations, 1,420 patients, consisting of 667 men and 753 women, were examined in this review. Using ImpactDose software, the estimated effective doses were 4.38 and $5.71 \mathrm{mSv}$ for males and females, respectively. The cancer risk was also estimated to be $36 \%$ higher in women than men, based on the PCXMC results. The most common findings in CT images were GGOs and consolidations. Most COVID-19 patients had multifocal and bilateral lesions; nevertheless, the amount of damage to the lung tissue changed rapidly. COVID-19 can target any lobe of the lungs, although lower lung lobes are mostly affected by COVID-19. It seems that lung damage observed in CT scans is more serious than clinical manifestations. Therefore, attention to CT images can significantly contribute to COVID19 diagnosis, as well as monitoring of patients during treatment.

Among patients with positive RT-PCR for COVID-19, but normal CT results, $93.4 \%$ were definitely in the early stages of the disease in the reviewed studies (no information for the rest) $(1,2,4,65,71)$. In other words, misdiagnosis by 
CT imaging might be related to the early stages of the disease when the lesions are not discernible in the lung tissue, even by HRCT scans. The estimated CT sensitivity was $90 \%$ in all studies reviewed. The highest and lowest CT sensitivities were $100 \%$ and $67 \%$, respectively.

In conclusion, for an optimized diagnosis of COVID-19, essential factors, including speed and accuracy of diagnosis, cost, ionizing radiation dose, and workload imposed on the healthcare system, should be considered. The findings of the present review revealed that diagnostic tools should be employed based on the time from the onset of symptom. In a study by Pan et al. (2), the sensitivity of CT was $81 \%$ in the first period of the study ( $2 \pm 2$ days after the symptom onset), which is in line with the present findings (79.1\% for 0 - 3 days after the symptom onset).

Within 0 - 3 days after the onset of symptoms (any symptom), the sensitivity of CT scan was estimated at $79.1 \%$. Although this sensitivity is higher than the total calculated sensitivity of RT-PCR (70\%), CT imaging showed the lowest sensitivity in this interval. Within 4 - 8 days after the onset of symptoms, the sensitivity of CT scan was estimated at $91.3 \%$, which seems to depend on the image acquisition parameters affecting image quality. Finally, nine days after the symptom onset, the sensitivity of CT scan was estimated at 95.1\%, regardless of imaging protocols. Therefore, a normal CT result in this interval indicates that suspected cases are not infected with COVID-19. However, the manifestation time and severity of COVID-19 were significantly different in various populations. Therefore, a combination of repeated swab tests and CT scanning can be used for highly suspected cases with negative results of RT-PCR.

\section{Acknowledgments}

This review article was financially supported by the Student Research Committee of Mashhad University of Medical Sciences, Mashhad, Iran (grant number 991161).

\section{Footnotes}

Authors' Contributions: Study concept and design: F.Gh., H.A-L, H.A., A.M., and M.N.; Acquisition of data: H.AL, H.A., F.Gh., and M.N.; Analysis and interpretation of data: H.A-L, F.Gh., H.A., M.N.; Drafting of the manuscript: S.S., H.AL, F.Gh., H.A., and M.N.; Critical revision of the manuscript for important intellectual content: A.M., B.A., H.A-L, and F.Gh.; Statistical analysis: H.A-L, H.A., and F.Gh.; Administrative, technical, and material support: H.A-L; and Study supervision: F.Gh.
Conflict of Interests: The authors declare that there is no conflict of interest and confirm the sole submission of this article to the "Iranian Journal of Radiology".

Funding/Support: This review article was financially supported by the Student Research Committee of Mashhad University of Medical Sciences (grant No.: 991161).

\section{References}

1. Bernheim A, Mei X, Huang M, Yang Y, Fayad ZA, Zhang N, et al. Chest CT findings in coronavirus disease-19 (COVID-19): Relationship to duration of infection. Radiology. 2020;295(3):200463. doi: 10.1148/radiol.2020200463. [PubMed: 32077789]. [PubMed Central: PMC7233369].

2. Pan F, Ye T, Sun P, Gui S, Liang B, Li L, et al. Time course of lung changes at chest CT during recovery from coronavirus disease 2019 (COVID19). Radiology. 2020;295(3):715-21. doi: 10.1148/radiol.2020200370. [PubMed: 32053470]. [PubMed Central: PMC7233367].

3. Ai T, Yang Z, Hou H, Zhan C, Chen C, Lv W, et al. Correlation of chest CT and RT-PCR testing for coronavirus disease 2019 (COVID19) in China: A report of 1014 cases. Radiology. 2020;296(2):E32-40. doi: 10.1148/radiol.2020200642. [PubMed: 32101510]. [PubMed Central: PMC7233399].

4. Xu YH, Dong JH, An WM, Lv XY, Yin XP, Zhang JZ, et al. Clinical and computed tomographic imaging features of novel coronavirus pneumonia caused by SARS-CoV-2.J Infect. 2020;80(4):394-400. doi: 10.1016/j.jinf.2020.02.017. [PubMed: 32109443]. [PubMed Central: PMC7102535].

5. Wu J, Wu X, Zeng W, Guo D, Fang Z, Chen L, et al. Chest CT findings in patients with coronavirus disease 2019 and its relationship with clinical features. Invest Radiol. 2020;55(5):257-61. doi: 10.1097/RLI.0000000000000670. [PubMed: 32091414]. [PubMed Central: PMC7147284].

6. Shi H, Han X, Jiang N, Cao Y, Alwalid O, Gu J, et al. Radiological findings from 81 patients with COVID-19 pneumonia in Wuhan, China: a descriptive study. Lancet Infect Dis. 2020;20(4):425-34. doi: 10.1016/S1473-3099(20)30086-4. [PubMed: 32105637]. [PubMed Central: PMC7159053].

7. Ng MY, Lee EYP, Yang J, Yang $\mathrm{F}$, Li X, Wang $\mathrm{H}$, et al. Imaging profile of the COVID-19 infection: Radiologic findings and literature review. Radiol Cardiothorac Imaging. 2020;2(1). e200034. doi: 10.1148/ryct.2020200034. [PubMed: 33778547]. [PubMed Central: PMC7233595].

8. Yang W, Cao Q, Qin L, Wang X, Cheng Z, Pan A, et al. Clinical characteristics and imaging manifestations of the 2019 novel coronavirus disease (COVID-19):A multi-center study in Wenzhou city, Zhejiang, China.JInfect. 2020;80(4):388-93. doi:10.1016/j.jinf.2020.02.016. [PubMed: 32112884]. [PubMed Central: PMC7102539].

9. Fang Y, Zhang H, Xie J, Lin M, Ying L, Pang P, et al. Sensitivity of chest CT for COVID-19: Comparison to RT-PCR. Radiology. 2020;296(2):E1157. doi: 10.1148/radiol.2020200432. [PubMed: 32073353]. [PubMed Central: PMC7233365].

10. Li X, Zeng W, Li X, Chen H, Shi L, Li X, et al. CT imaging changes of corona virus disease 2019(COVID-19): a multi-center study in Southwest China. J Transl Med. 2020;18(1):154. doi: 10.1186/s12967-020-02324w. [PubMed: 32252784]. [PubMed Central: PMC7132551].

11. Pan Y, Guan H, Zhou S, Wang Y, Li Q, Zhu T, et al. Initial CT findings and temporal changes in patients with the novel coronavirus pneumonia (2019-nCoV): a study of 63 patients in Wuhan, China. Eur 
Radiol. 2020;30(6):3306-9. doi: 10.1007/s00330-020-06731-x. [PubMed: 32055945]. [PubMed Central: PMC7087663].

12. Zhao W, Zhong Z, Xie X, Yu Q, Liu J. Relation between chest CT findings and clinical conditions of coronavirus disease (COVID-19) pneumonia: A multicenter study. AJR Am J Roentgenol. 2020;214(5):1072-7. doi: 10.2214/AJR.20.22976. [PubMed: 32125873].

13. Wong KT, Antonio GE, Hui DS, Lee N, Yuen EH, Wu A, et al. Thin-section CT of severe acute respiratory syndrome: Evaluation of 73 patients exposed to or with the disease. Radiology. 2003;228(2):395-400. doi: 10.1148/radiol.2283030541. [PubMed: 12738877].

14. Liu L, Gao J, Hu W, Zhang X, Guo L, Liu C, et al. Clinical characteristics of 51 patients discharged from hospital with COVID-19 in Chongqing, China. MedRxiv. 2020. doi: 10.1101/2020.02.20.20025536.

15. Cheng Z, Lu Y, Cao Q, Qin L, Pan Z, Yan F, et al. Clinical features and chest CT manifestations of coronavirus disease 2019 (COVID19) in a single-center study in Shanghai, China. AJR Am J Roentgenol. 2020;215(1):121-6. doi: 10.2214/AJR.20.22959. [PubMed: 32174128].

16. Qian GQ, Yang NB, Ding F, Ma AHY, Wang ZY, Shen YF, et al. Epidemiologic and clinical characteristics of 91 hospitalized patients with COVID-19 in Zhejiang, China: A retrospective, multi-centre case series. QJM. 2020;113(7):474-81. doi: 10.1093/qjmed/hcaa089. [PubMed: 32181807]. [PubMed Central: PMC7184349].

17. Han R, Huang L, Jiang H, Dong J, Peng H, Zhang D. Early clinical and CT manifestations of coronavirus disease 2019 (COVID-19) pneumonia. AJR Am J Roentgenol. 2020;215(2):338-43. doi: 10.2214/AJR.20.22961. [PubMed: 32181672].

18. Huang C, Wang Y, Li X, Ren L, Zhao J, Hu Y, et al. Clinical features of patients infected with 2019 novel coronavirus in Wuhan, China. Lancet. 2020;395(10223):497-506. doi: 10.1016/S0140-6736(20)30183-5. [PubMed: 31986264]. [PubMed Central: PMC7159299].

19. Zhu T, Wang Y, Zhou S, Zhang N, Xia L. A comparative study of chest computed tomography features in young and older adults with corona virus disease (COVID-19). J Thorac Imaging. 2020;35(4):W97W101. doi: 10.1097/RTI.0000000000000513. [PubMed: 32235187]. [PubMed Central: PMC7253040].

20. Gao L, Zhang J. Pulmonary high-resolution computed tomography (HRCT) findings of patients with early-stage coronavirus disease 2019 (COVID-19) in Hangzhou, China. Med Sci Monit. 2020;26. e923885. doi: 10.12659/MSM.923885. [PubMed: 32246819]. [PubMed Central: PMC7156878].

21. Xu T, Chen C, Zhu Z, Cui M, Chen C, Dai H, et al. Clinical features and dynamics of viral load in imported and non-imported patients with COVID-19. Int J Infect Dis. 2020;94:68-71. doi: 10.1016/j.ijid.2020.03.022. [PubMed: 32179140]. [PubMed Central: PMC7270709].

22. Li K, Wu J, Wu F, Guo D, Chen L, Fang Z, et al. The clinical and chest CT features associated with severe and critical COVID-19 pneumonia. Invest Radiol. 2020;55(6):327-31. doi: 10.1097/RLI.0000000000000672. [PubMed: 32118615]. [PubMed Central: PMC7147273].

23. Song F, Shi N, Shan F, Zhang Z, Shen J, Lu H, et al. Emerging 2019 novel coronavirus (2019-nCoV) pneumonia. Radiology. 2020;297(3). E346. doi: 10.1148/radiol.2020209021. [PubMed: 33196374].

24. Zheng C, Wang J, Guo H, Lu Z, Ma Y, Zhu Y, et al. Risk-adapted treatment strategy for COVID-19 patients. Int J Infect Dis. 2020;94:74-7. doi: 10.1016/j.ijid.2020.03.047. [PubMed: 32229257]. [PubMed Central: PMC7270846].

25. Zhu W, Xie K, Lu H, Xu L, Zhou S, Fang S. Initial clinical features of suspected coronavirus disease 2019 in two emergency departments outside of Hubei, China. J Med Virol. 2020;92(9):152532. doi: 10.1002/jmv.25763. [PubMed: 32167181]. [PubMed Central: PMC7228360].

26. Xiong Y, Sun D, Liu Y, Fan Y, Zhao L, Li X, et al. Clinical and highresolution CT features of the COVID-19 infection: Comparison of the initial and follow-up changes. Invest Radiol. 2020;55(6):332-9. doi: 10.1097/RLI.0000000000000674. [PubMed: 32134800]. [PubMed Central: PMC7147282].

27. Zhou Z, Guo D, Li C, Fang Z, Chen L, Yang R, et al. Coronavirus disease 2019: initial chest CT findings. Eur Radiol. 2020;30(8):4398-406. doi: 10.1007/s00330-020-06816-7. [PubMed: 32211963]. [PubMed Central: PMC7095437].

28. Li Y, Xia L. Coronavirus disease 2019 (COVID-19): Role of chest CT in diagnosis and management. AJR Am J Roentgenol. 2020;214(6):1280-6. doi: 10.2214/AJR.20.22954. [PubMed: 32130038].

29. Yang S, Shi Y, Lu H, Xu J, Li F, Qian Z, et al. Clinical and CT features of early stage patients with COVID-19: a retrospective analysis of imported cases in Shanghai, China. Eur Respir J. 2020;55(4). doi: 10.1183/13993003.00407-2020. [PubMed: 32217649]. [PubMed Central: PMC7098483].

30. Long C, Xu H, Shen Q, Zhang X, Fan B, Wang C, et al. Diagnosis of the Coronavirus disease (COVID-19): rRT-PCR or CT? Eur J Radiol. 2020;126:108961. doi: 10.1016/j.ejrad.2020.108961. [PubMed: 32229322]. [PubMed Central: PMC7102545].

31. Himoto Y, Sakata A, Kirita M, Hiroi T, Kobayashi KI, Kubo K, et al. Diagnostic performance of chest CT to differentiate COVID-19 pneumonia in non-high-epidemic area in Japan.Jpn J Radiol.2020;38(5):400-6. doi: 10.1007/s11604-020-00958-w. [PubMed: 32232648]. [PubMed Central: PMC7105967].

32. Bai HX, Hsieh B, Xiong Z, Halsey K, Choi JW, Tran TML, et al. Performance of radiologists in differentiating COVID-19 from non-COVID19 viral pneumonia at chest CT. Radiology. 2020;296(2):E46-54. doi: 10.1148/radiol.2020200823. [PubMed: 32155105]. [PubMed Central: PMC7233414].

33. Dai WC, Zhang HW, Yu J, Xu HJ, Chen H, Luo SP, et al. CT imaging and differential diagnosis of COVID-19. Can Assoc Radiol J. 2020;71(2):195200. doi: 10.1177/0846537120913033. [PubMed: 32129670]. [PubMed Central: PMC7140975].

34. Wong HYF, Lam HYS, Fong AH, Leung ST, Chin TW, Lo CSY, et al. Frequency and distribution of chest radiographic findings in patients positive for COVID-19. Radiology. 2020;296(2):E72-8. doi: 10.1148/radiol.2020201160. [PubMed: 32216717]. [PubMed Central: PMC7233401].

35. Liu KC, Xu P, Lv WF, Qiu XH, Yao JL, Gu JF, et al. CT manifestations of coronavirus disease-2019: A retrospective analysis of 73 cases by disease severity. Eur J Radiol. 2020;126:108941. doi 10.1016/j.ejrad.2020.108941. [PubMed: 32193037]. [PubMed Central: PMC7118536].

36. Li K, Fang Y, Li W, Pan C, Qin P, Zhong Y, et al. CT image visual quantitative evaluation and clinical classification of coronavirus disease (COVID-19). Eur Radiol. 2020;30(8):4407-16. doi: 10.1007/s00330-02006817-6. [PubMed: 32215691]. [PubMed Central: PMC7095246].

37. Kim ES, Chin BS, Kang CK, Kim NJ, Kang YM, Choi JP, et al. Clinical course and outcomes of patients with severe acute respiratory syndrome coronavirus 2 infection: a preliminary report of the first 28 patients from the Korean cohort study on COVID-19. J Korean Med Sci. 2020;35(13). e142. doi: 10.3346/jkms.2020.35.e142. [PubMed: 32242348]. [PubMed Central: PMC7131901].

38. Li N, Han L, Peng M, Lv Y, Ouyang Y, Liu K, et al. Maternal and neonatal outcomes of pregnant women with coronavirus disease 2019 (COVID19) pneumonia: A case-control study. Clin Infect Dis. 2020;71(16):203541. doi: 10.1093/cid/ciaa352. [PubMed: 32249918]. [PubMed Central: PMC7184430].

39. Han X, Cao Y, Jiang N, Chen Y, Alwalid O, Zhang X, et al. Novel coronavirus disease 2019 (COVID-19) pneumonia progression course in 17 discharged patients: Comparison of clinical and thin-section computed tomography features during recovery. Clin Infect Dis. 2020;71(15):723-31. doi: 10.1093/cid/ciaa271. [PubMed: 32227091]. [PubMed Central: PMC7184369].

40. Chen Z, Fan H, Cai J, Li Y, Wu B, Hou Y, et al. High-resolution 
computed tomography manifestations of COVID-19 infections in patients of different ages. Eur J Radiol. 2020;126:108972. doi: 10.1016/j.ejrad.2020.108972. [PubMed: 32240913]. [PubMed Central: PMC7102649].

41. Wu J, Liu J, Zhao X, Liu C, Wang W, Wang D, et al. Clinical characteristics of imported cases of coronavirus disease 2019 (COVID19) in Jiangsu Province: A multicenter descriptive study. Clin Infect Dis. 2020;71(15):706-12. doi: 10.1093/cid/ciaa199. [PubMed: 32109279]. [PubMed Central: PMC7108195].

42. Wang L, Gao YH, Lou LL, Zhang GJ. The clinical dynamics of 18 cases of COVID-19 outside of Wuhan, China. Eur Respir J. 2020;55(4). doi: 10.1183/13993003.00398-2020. [PubMed: 32139464]. [PubMed Central: PMC7098482].

43. Zhang X, Cai H, Hu J, Lian J, Gu J, Zhang S, et al. Epidemiological, clinical characteristics of cases of SARS-CoV-2 infection with abnormal imaging findings. Int J Infect Dis. 2020;94:81-7. doi: 10.1016/j.ijid.2020.03.040. [PubMed: 32205284]. [PubMed Central: PMC7270493]

44. Iwasawa T, Sato M, Yamaya T, Sato Y, Uchida Y, Kitamura H, et al. Ultra-high-resolution computed tomography can demonstrate alveolar collapse in novel coronavirus (COVID-19) pneumonia. Jpn J Radiol. 2020;38(5):394-8. doi: 10.1007/s11604-020-00956-y. [PubMed: 32236856]. [PubMed Central: PMC7110271].

45. Caruso D, Zerunian M, Polici M, Pucciarelli F, Polidori T, Rucci C, et al. Chest CT features of COVID-19 in Rome, Italy. Radiol ogy. 2020;296(2):E79-85. doi: 10.1148/radiol.2020201237. [PubMed: 32243238]. [PubMed Central: PMC7194020].

46. Zhao D, Yao F, Wang L, Zheng L, Gao Y, Ye J, et al. A comparative study on the clinical features of coronavirus 2019 (COVID-19) pneumonia with other Pneumonias. Clin Infect Dis. 2020;71(15):75661. doi: 10.1093/cid/ciaa247. [PubMed: 32161968]. [PubMed Central: PMC7108162].

47. Chen N, Zhou M, Dong X, Qu J, Gong F, Han Y, et al. Epidemiological and clinical characteristics of 99 cases of 2019 novel coronavirus pneumonia in Wuhan, China: a descriptive study. Lancet 2020;395(10223):507-13. doi: 10.1016/S0140-6736(20)30211-7. [PubMed: 32007143]. [PubMed Central: PMC7135076].

48. Bai Y, Yao L, Wei T, Tian F, Jin DY, Chen L, et al. Presumed asymptomatic carrier transmission of COVID-19. JAMA. 2020;323(14):1406-7. doi: 10.1001/jama.2020.2565. [PubMed: 32083643]. [PubMed Central: PMC7042844].

49. Wan S, Xiang Y, Fang W, Zheng Y, Li B, Hu Y, et al. Clinical features and treatment of COVID-19 patients in northeast Chongqing. J Med Virol.2020;92(7):797-806. doi: 10.1002/jmv.25783. [PubMed: 32198776] [PubMed Central: PMC7228368].

50. Lin C, Ding Y, Xie B, Sun Z, Li X, Chen Z, et al. Asymptomatic novel coronavirus pneumonia patient outside Wuhan: The value of $\mathrm{CT}$ images in the course of the disease. Clin Imaging. 2020;63:7-9. doi 10.1016/j.clinimag.2020.02.008. [PubMed: 32120312]. [PubMed Central: PMC7125867]

51. Zhou S, Wang Y, Zhu T, Xia L. CT features of coronavirus disease 2019 (COVID-19) Pneumonia in 62 patients in Wuhan, China. AJR Am J Roentgenol. 2020;214(6):1287-94. doi: 10.2214/AJR.20.22975. [PubMed: 32134681].

52. Wang Y, Dong C, Hu Y, Li C, Ren Q, Zhang X, et al. Temporal changes of CT findings in 90 patients with COVID-19 pneumonia: A longitudinal study. Radiology. 2020;296(2):E55-64. doi: 10.1148/radiol.2020200843. [PubMed: 32191587]. [PubMed Central: PMC7233482].

53. Du Y, Tu L, Zhu P, Mu M, Wang R, Yang P, et al. Clinical features of 85 fatal cases of COVID-19 from Wuhan. A retrospective observational study. Am J Respir Crit Care Med. 2020;201(11):1372-9. doi: 10.1164/rccm.202003-05430C. [PubMed: 32242738]. [PubMed Central: PMC7258652].
54. Huang G, Gong T, Wang G, Wang J, Guo X, Cai E, et al. Timely diagnosis and treatment shortens the time to resolution of coronavirus disease (COVID-19) pneumonia and lowers the highest and last CT scores from sequential chest CT. AJR Am J Roentgenol. 2020;215(2):367-73. doi: 10.2214/AJR.20.23078. [PubMed: 32223665].

55. Wang K, Kang S, Tian R, Zhang X, Zhang X, Wang Y. Imaging manifestations and diagnostic value of chest $\mathrm{CT}$ of coronavirus disease 2019 (COVID-19) in the Xiaogan area. Clin Radiol. 2020;75(5):341-7. doi: 10.1016/j.crad.2020.03.004. [PubMed: 32216961]. [PubMed Central: PMC7118637].

56. Xu X, Yu C, Qu J, Zhang L, Jiang S, Huang D, et al. Imaging and clinical features of patients with 2019 novel coronavirus SARS-CoV-2. Eur J Nucl Med Mol Imaging. 2020;47(5):1275-80. doi: 10.1007/s00259-020-047359. [PubMed: 32107577]. [PubMed Central: PMC7080117].

57. Chung M, Bernheim A, Mei X, Zhang N, Huang M, Zeng X, et al. CT imaging features of 2019 novel coronavirus (2019-nCoV). Radiology. 2020;295(1):202-7. doi: 10.1148/radiol.2020200230. [PubMed: 32017661]. [PubMed Central: PMC7194022].

58. Liu D, Li L, Wu X, Zheng D, Wang J, Yang L, et al. Pregnancy and perinatal outcomes of women with coronavirus disease (COVID-19) pneumonia: A preliminary analysis. AJR Am J Roentgenol. 2020;215(1):127-32. doi: 10.2214/AJR.20.23072. [PubMed: 32186894].

59. Cavagna E, Muratore F, Ferrari F. Pulmonary thromboembolism in COVID-19: Venous thromboembolism or arterial thrombosis? Radiol Cardiothorac Imaging. 2020;2(4). e200289. doi: 10.1148/ryct.2020200289. [PubMed: 33778609]. [PubMed Central: PMC7350032].

60. Kaminetzky M, Moore W, Fansiwala K, Babb JS, Kaminetzky D, Horwitz LI, et al. Pulmonary embolism at CT pulmonary angiography in patients with COVID-19. Radiol Cardiothorac Imaging. 2020;2(4). e200308. doi: 10.1148/ryct.2020200308. [PubMed: 33778610]. [PubMed Central: PMC7336753].

61. Zotzmann V, Lang CN, Bamberg F, Bode C, Staudacher DL. Are subpleural consolidations indicators for segmental pulmonary embolism in COVID-19? Intensive Care Med.2020;46(6):1109-10. doi:10.1007/s00134020-06044-z. [PubMed: 32328727]. [PubMed Central: PMC7178099]

62. Zhang JJ, Dong X, Cao YY, Yuan YD, Yang YB, Yan YQ, et al. Clinical characteristics of 140 patients infected with SARS-CoV-2 in Wuhan, China. Allergy. 2020;75(7):1730-41. doi: 10.1111/all.14238. [PubMed: 32077115].

63. Poon LC, Yang H, Dumont S, Lee JCS, Copel JA, Danneels L, et al. ISUOG Interim Guidance on coronavirus disease 2019 (COVID-19) during pregnancy and puerperium: information for healthcare professionals - an update. Ultrasound Obstet Gynecol. 2020;55(6):84862. doi: 10.1002/uog.22061. [PubMed: 32356590]. [PubMed Central: PMC7267438].

64. Xie X, Zhong Z, Zhao W, Zheng C, Wang F, Liu J. Chest CT for typical coronavirus disease 2019 (COVID-19) pneumonia: Relationship to negative RT-PCR testing. Radiology. 2020;296(2):E41-5. doi: 10.1148/radiol.2020200343. [PubMed: 32049601]. [PubMed Central: PMC7233363].

65. Guan W, Ni Z, Hu Y, Liang W, Ou C, He J, et al. Clinical characteristics of 2019 novel coronavirus infection in China. N Engl J Med. 2020;42(1):914. doi: 10.1101/2020.02.06.20020974.

66. Guan CS, Lv ZB, Yan S, Du YN, Chen H, Wei LG, et al. Imaging features of coronavirus disease 2019 (COVID-19): Evaluation on Thin-Section CT. Acad Radiol. 2020;27(5):609-13. doi: 10.1016/j.acra.2020.03.002. [PubMed: 32204990]. [PubMed Central: PMC7156158].

67. Chen H, Guo J, Wang C, Luo F, Yu X, Zhang W, et al. Clinical characteristics and intrauterine vertical transmission potential of COVID19 infection in nine pregnant women: A retrospective review of medical records. Lancet. 2020;395(10226):809-15. doi: 10.1016/S01406736(20)30360-3. [PubMed: 32151335]. [PubMed Central: PMC7159281]. 68. Hu Z, Song C, Xu C, Jin G, Chen Y, Xu X, et al. Clinical characteris- 
tics of 24 asymptomatic infections with COVID-19 screened among close contacts in Nanjing, China. Sci China Life Sci. 2020;63(5):706-11. doi: 10.1007/s11427-020-1661-4. [PubMed: 32146694]. [PubMed Central: PMC7088568].

69. Gunderman RB. Knowing and being in pediatric radiology. Pediatr Radiol. 2020;50(1):1-2. doi: 10.1007/s00247-019-04573-4. [PubMed: 31901986].

70. Lei P, Fan B, Mao J, Wei J, Wang P. The progression of computed tomographic (CT) images in patients with coronavirus disease (COVID-19) pneumonia: Running title: The CT progression of COVID-19 pneumonia.JInfect. 2020;80(6):e30-1. doi:10.1016/j.jinf.2020.03.020.[PubMed: 32205140]. [PubMed Central: PMC7195117].

71. Wang X, Fang J, Zhu Y, Chen L, Ding F, Zhou R, et al. Clinical characteristics of non-critically ill patients with novel coronavirus infection (COVID-19) in a Fangcang Hospital. Clin Microbiol Infect. 2020;26(8):1063-8. doi: 10.1016/j.cmi.2020.03.032. [PubMed: 32251842]. [PubMed Central: PMC7195539].

72. Li Q, Guan X, Wu P, Wang X, Zhou L, Tong Y, et al. Early transmission dynamics in Wuhan, China, of novel coronavirus-infected pneumonia. N Engl J Med. 2020;382(13):1199-207. doi:10.1056/NEJMoa2001316.

73. Chu DKW, Pan Y, Cheng SMS, Hui KPY, Krishnan P, Liu Y, et al. Molecular diagnosis of a novel coronavirus (2019-nCoV) causing an outbreak of pneumonia. Clin Chem. 2020;66(4):549-55. doi: 10.1093/clinchem/hvaa029. [PubMed: 32031583]. [PubMed Central: PMC7108203].

74. Chau TN, Lee PO, Choi KW, Lee CM, Ma KF, Tsang TY, et al. Value of initial chest radiographs for predicting clinical outcomes in patients with severe acute respiratory syndrome. Am J Med. 2004;117(4):24954. doi: 10.1016/j.amjmed.2004.03.020. [PubMed:15308434].[PubMed Central: PMC7093886].

75. Mettler FJ, Huda W, Yoshizumi TT, Mahesh M. Effective doses in radiology and diagnostic nuclear medicine: a catalog. Radiology. 2008;248(1):254-63. doi: 10.1148/radiol.2481071451. [PubMed: 18566177].

76. Wang S, Kang B, Ma J, Zeng X, Xiao M, Guo J, et al. A deep learning algorithm using CT images to screen for corona virus disease (COVID-19). MedRxiv. 2020. doi:10.1101/2020.02.14.20023028.

77. Beik J, Jafariyan M, Montazerabadi A, Ghadimi-Daresajini A, Tarighi $\mathrm{P}$, Mahmoudabadi A, et al. The benefits of folic acid-modified gold nanoparticles in CT-based molecular imaging: Radiation dose reduction and image contrast enhancement. ArtifCells Nanomed Biotechnol. 2018;46(8):1993-2001. doi: 10.1080/21691401.2017.1408019. [PubMed: 29233015].

78. Tapiovaara M S. PCXMC, A Monte Carlo program for calculating patient doses in medical $x$-ray examinations. Helsinki/Finland: STUK; 2008.

79. Council NR. Health risks from exposure to low levels of ionizing radiation: BEIR VII phase 2. National Academies Press; 2006.

80. Hsieh J. Computed tomography: Principles, design, artifacts, and recent advances. SPIE press; 2015.

81. Taghavi H, Bakhshandeh M, Montazerabadi A, Nazari Moghadam H, Mazloom Shahri SB, Keshtkar M. Comparison of gold nanoparticles and iodinated contrast media in radiation dose reduction and contrast enhancement in computed tomography. Iran J Radiol. 2020;17(1) doi: 10.5812/iranjradiol.92446. 